\title{
SUPRESSÃO DE PLANTAS ESPONTÂNEAS POR ADUBOS VERDES EM ÁREA DE CULTIVO ORGÂNICO
}

\section{SUPPRESSION OF SPONTANEOUS PLANTS BY GREEN FERTILIZERS IN AN ORGANIC CULTIVATION AREA}

\author{
Jason Mateus Queiroz Silva ${ }^{1}$ \\ Natalia Machado Lacerda ${ }^{2}$ \\ João José Costa Silva ${ }^{3}$ \\ Endrius Gabriel de Oliveira da Silva ${ }^{4}$ \\ Carlos Francisco Salgado Barroso 5 \\ Lelisângela Carvalho da Silva ${ }^{6}$
}

\begin{abstract}
RESUMO: Este trabalho teve como objetivo avaliar o efeito de três adubos verdes na supressão de plantas espontâneas em uma área de cultivo orgânico. $\mathrm{O}$ experimento foi realizado em uma propriedade particular localizada no município de Rorainópolis-Roraima, o delineamento experimental em blocos ao acaso, utilizando espécies de crotalária (Crotalaria spectabilis e Crotalaria ochroleuca), feijão guandu (Cajanus cajan) e uma testemunha sem leguminosa, com quatro repetições. Foi usado como variável: massa seca e fresca e a densidade de plantas espontâneas aos 36 e 60 dias após a semeadura das leguminosas (DAS). A massa seca e fresca das plantas espontâneas foi inferior a testemunha nos dois períodos avaliados, com destaque para Crotalaria spectabilis e Crotalaria ochroleuca, que obtiveram melhores resultados na redução de biomassa das plantas espontâneas. Quanto a redução da densidade de plantas espontâneas, Crotalaria spectabilis apresentou melhor eficiência, comparada aos outros tratamentos. Assim, para este experimento, é recomendado o uso de adubos verdes, para um controle eficiente de plantas espontâneas.
\end{abstract}

${ }^{1}$ Acadêmico de Agronomia da Universidade Estadual de Roraima (UERR). E-mail: jason.queiroz1999@gmail.com;

${ }^{2}$ Acadêmica de Engenharia Florestal (UERR). E-mail: lacerrda33@gmail.com;

${ }^{3}$ Professor da UERR. E-mail: joao@uerr.edu.br;

${ }^{4}$ Acadêmico de Agronomia (UERR). E-mail: endrius2001gabriel@gmail.com;

${ }^{5}$ Bolsista do Núcleo de Estudo em Agroecologia e Produção Orgânica da UERR. E-mail: carlossb10m@live.com;

${ }^{6}$ Professora da UERR. E-mail: lelisangela@uerr.edu.br. 
Palavras-chave: Agroecossistemas, Supressão, Crotalárias, Feijão guandu.

\begin{abstract}
The objective of this work was to evaluate the effect of three green manures on the suppression of spontaneous plants in an area of organic cultivation. The experiment was carried out in a private property located in the municipality of Rorainópolis-Roraima, in a randomized block design, using crotalaria species (Crotalaria spectabilis and Crotalaria ochroleuca), pigeon pea (Cajanus cajan) and a control without the presence of green fertilizer, with four replicates. Fresh and dry weight and density of the spontaneous plant were used as response variables at 36 and 60 days after sowing of legumes (DAS). The dry and fresh mass of the spontaneous plants was lower than the control in the two evaluated periods, especially Crotalaria spectabilis and Crotalaria ochroleuca, which obtained good results in the reduction of biomass of the spontaneous plants. As for the reduction of spontaneous plant density, Crotalaria spectabilis presented an excellent efficiency compared to the other treatments. Thus, for this experiment, it is recommended the use of green fertilizers, for an efficient control of spontaneous plants.
\end{abstract}

Key-words: Agroecosystems, Suppression, Crotalaria, Pigeon peas.

\title{
INTRODUÇÃO
}

A agroecologia possibilita opções que vão mais adiante das práticas e organização de agroecossistemas com baixa dependência de agroquímicos e energia externa. Porém, a substituição de insumos que não provoquem consequências negativas ao ambiente é uma das principais etapas para a instalação, início e sustentabilidade desses sistemas (ALTIERI; NICHOLLS, 2005).

O controle de plantas espontâneas é uma das principais dificuldades na busca pela sustentabilidade de sistemas agrícolas que possuem base ecológica, apesar de apresentar um valor biológico em alguns agroecossistemas, também pode ser prejudicial ao rendimento da cultura principal. Portanto, é viável a busca de soluções como adubos verdes, considerados como forma eficiente na busca da sustentabilidade dos solos, onde protegem e disponibilizam nutrientes ao solo, e também podem suprimir plantas espontâneas (Do NASCIMENTO; De MATTOS, 2008). 
Para o controle de plantas espontâneas é necessário combinar diferentes práticas que visem o aproveitamento dos recursos disponíveis de modo a proporcionar maior eficácia, redução dos custos, máxima segurança para o homem e mínima contaminação ou alteração do ambiente. Enquanto que, o manejo de plantas espontâneas refere-se à combinação de métodos de controle que quando associados ao controle de pragas, doenças e erosão, constitui o manejo integrado. Os principais métodos de controle de plantas espontâneas são o preventivo, o biológico, controle químico, o cultural e o mecânico ou físico (COBUCCI, 2004).

O método preventivo baseia-se no conhecimento dos processos básicos de reprodução e disseminação das plantas espontâneas, a fim de interromper seu ciclo de multiplicação e disseminação. Já o método de controle biológico é realizado por agentes biológicos como insetos, ácaros e fungos, os quais são muitos seletivos em sua ação de controle e pode restringir-se apenas a uma única espécie, sendo considerado um método apropriado para lavouras de menores dimensões (BORÉM; RANGEL, 2015).

De acordo com Lorenzi (2014), o controle químico consiste no uso de produtos químicos (herbicidas) com capacidade de matar plantas por contato ou por meios mais complexos, após a absorção do produto pela planta. Já o controle cultural é feito utilizando práticas comuns, como rotação de cultura, uso de coberturas verdes e variação de espaçamento da cultura. Logo o controle mecânico ou físico faz uso de práticas de eliminação de plantas espontâneas através do efeito físico-mecânico, como o arranquio manual (monda), a capina manual, a roçada, a inundação, a queimada, cultivo mecanizado e a cobertura morta.

De Almeida (1991) relata que a supressão desempenhada por alguns adubos verdes sobre plantas espontâneas pode ser devido aos efeitos alelopáticos. No entanto, esse efeito pode ser atribuído à barreira física exercida pelos mesmos, dificultando a disponibilidade de água, luz, gases e nutrientes no solo (FAVERO et al., 2001).

Conforme Maldonado et al. (2001), o emprego de adubos verdes pode ser eficiente na redução da população de plantas espontâneas, diminuindo expressivamente a sua infestação, possibilitando ser usado para o manejo integrado dessas plantas. Bond; Grundy (2001) comentam que coberturas de solo, vivas ou não, são mais eficientes na prevenção de germinação de sementes de plantas espontâneas e emergência de plântulas que na supressão de plantas já estabelecidas. 
Nesse contexto, o presente trabalho teve como objetivo avaliar a capacidade de três leguminosa: (Cajanus cajan, Crotalaria spectabilis e Crotalaria ochroleuca), de suprimir a vegetação espontânea em área de cultivo orgânico.

\section{MATERIAL E MÉTODOS}

O estudo foi conduzido na unidade demonstrativa em Agroecologia da Universidade Estadual de Roraima/UERR em parceria com produtor orgânico, localizada na vicinal 19 em área de assentamento rural no munícipio de Rorainópolis, região sul do estado de Roraima $\left(00^{\circ} 51^{\prime} 31^{\prime \prime} \mathrm{S}\right.$ e 60²1'49" W, à altitude de $\left.107 \mathrm{~m}\right)$. A área de estudo estava com a presença de vegetação espontânea composta principalmente por gramíneas, por um período superior a dois anos, tendo sido anteriormente cultivada com melancia. $\mathrm{O}$ clima da região é classificado como Af (ALVARES, 2013), com precipitação média anual de 2200-2500 mm sem presença de estação seca.

O relevo caracteriza-se como suave-ondulado, e o solo classificado como Latossolo, sendo sua vegetação nativa composta por floresta ombrófila densa. A análise química preliminar da camada de $0-0,20 \mathrm{~m}$ do solo da área experimental apresentou os seguintes resultados: pHH2O: 5,3; $\mathrm{P}\left(\right.$ Mehlich1): 0,9 mg. $\mathrm{dm}^{-3}$; $\mathrm{K}:$ 0,38; $\mathrm{Ca}+\mathrm{Mg}: 24,0$ e $\mathrm{Al}:$ 4,0; com todas unidades expressas em cmolc. $\mathrm{dm}^{-3}$. As etapas de preparo do solo foram divididas em aração e gradagem com posterior calagem realizada em área total a lanço manual e incorporação mecânica de 3,5 Mg.ha ${ }^{-1}$ de calcário dolomítico (PRNT=82\%), 30 dias antes do semeio. A fosfatagem foi dívida em duas operações sendo uma realizada em área total e a outra localizada na linha de cultivo.

Para analisar a supressão de plantas espontâneas foi realizado o delineamento experimental em blocos ao acaso com três tratamentos mais uma testemunha sem leguminosas com 4 repetições, em que os tratamentos foram representados pelas leguminosas: Cajanus cajan, Crotalaria ochroleuca e Crotalaria spectabilis. As parcelas experimentais foram demarcadas nas seguintes dimensões 5,0 X 26,0 m, totalizando $130 \mathrm{~m}^{2}$, cada uma delas com 10 linhas de cultivo e espaçadas entre si por 0,50 m para todas as leguminosas, já o semeio, foi realizado manualmente na linha de cultivo seguindo as recomendações de acordo com cada cultivar: Cajanus cajan (10 sementes.metro $\left.^{-1}\right)$, Crotalaria spectabilis (30 sementes.metro $^{-1}$ ) e Crotalaria ochroleuca (40 sementes.metro ${ }^{-1}$ ) com profundidade de 
plantio adotada para todos os tratamentos de $0,02 \mathrm{~m}$. As leguminosas foram semeadas e acompanhadas até a fase de florescimento.

As plantas espontâneas da área experimental foram amostradas nos tratamentos e na testemunha, para avaliação da massa seca, massa fresca e densidade de plantas espontâneas em cada parcela, em dois períodos: 36 e 60 dias após semeadura das leguminosas (DAS). A amostragem das plantas espontâneas nas parcelas foi realizada com auxílio de um retângulo de ferro, com dimensões de 0,435 x 0,28 $\mathrm{m}\left(0,12 \mathrm{~m}^{2}\right.$ de área), o qual foi lançado de forma aleatória dentro da área útil de cada parcela, esse processo foi repetido quatro vezes em cada parcela.

Esse posicionamento foi alterado também em cada período de avaliação, para não repetir o local anteriormente amostrado. As plantas contidas no retângulo foram coletadas no período da manhã, contadas e depositadas em sacos de papel, após a coleta foi determinado a massa fresca e colocadas em estufa de ventilação forçada a $65^{\circ} \mathrm{C}$, por 72 horas, até atingirem massa constante e em seguida, pesadas novamente para determinação da massa seca.

Os resultados da densidade, massa seca e fresca da vegetação espontânea foram expressos em número de plantas por $\mathrm{m}^{-2}$ e g. $\mathrm{m}^{-2}$, respectivamente. Os dados referentes a cada período de avaliação foram submetidos à análise de variância. Sendo as médias comparadas pelo teste de Tukey a $5 \%$, utilizando-se o software estatístico (TEAM, Rstudio. 2015).

\section{RESULTADOS}

Houve diferenças significativas na produção de massa seca das plantas espontâneas aos 36 DAS e aos 60 DAS (Tabela 1). Aos 36 DAS todas as leguminosas apresentaram eficiência na supressão de plantas espontâneas em comparação com a testemunha (Tabela 1), com destaque para C. ochroleuca, que apresentou uma média de 85,9 (g.m $\mathrm{m}^{-2}$ ) de massa seca de plantas espontâneas, o que pode ser atribuído a sua velocidade de crescimento proporcionando o sombreamento mais rápido.

Aos 60 DAS a $C$. spectabilis e a $C$. ochroleuca, apresentaram maiores capacidades de supressão em função da massa seca de plantas espontâneas, onde as parcelas com $C$. spectabilis apresentaram menor acúmulo de massa seca de plantas espontâneas tendo em média $31,2\left(\right.$ g.m $\left.{ }^{-2}\right)$, correspondendo a uma taxa de $73,3 \%$ de redução da massa seca de plantas espontâneas ao longo do período estudado, e a C. ochroleuca 117,1 (g.m $\left.{ }^{-2}\right)$, 
determinando que ao longo do tempo a $C$. spectabilis pode apresentar melhor desempenho na supressão de plantas espontâneas nas condições estudas.

Observando a leguminosa C. cajan aos 60 DAS (tabela 1), é possível notar que ela se mostra inferior em relação as demais, porém ainda assim apresenta melhor desempenho que a testemunha. Entretanto, aos 36 DAS apresenta uma média de 106,7 (g.m ${ }^{-2}$ ) que é estatisticamente igual as demais leguminosas em relação a massa seca de plantas espontâneas.

Tabela 1. Efeito dos tratamentos utilizados, na redução de Densidade, Massa seca e Massa fresca de plantas espontâneas em dois períodos, 36 e 60 dias após a semeadura (DAS).

\begin{tabular}{|c|c|c|c|c|c|c|}
\hline \multirow{2}{*}{ Tratamentos } & \multicolumn{2}{|c|}{ Densidade (plantas $\mathrm{m}^{-2}$ ) } & \multicolumn{2}{|c|}{ Massa seca $\left(\mathrm{g} \mathrm{m}^{-2}\right)$} & \multicolumn{2}{|c|}{ Massa fresca $\left(\mathrm{g} \mathrm{m}^{-2}\right)$} \\
\hline & 36 DAS & 60 DAS & 36 DAS & $60 \mathrm{DAS}$ & $36 \mathrm{DAS}$ & 60 DAS \\
\hline C. spectabilis & $205,7 \mathrm{a}$ & $78,1 \mathrm{a}$ & $117,1 \mathrm{a}$ & $31,2 \mathrm{a}$ & $794,3 \mathrm{a}$ & $106,8 \mathrm{a}$ \\
\hline C. ochroleuca & $230,2 \mathrm{a}$ & $282,8 b$ & $85,9 \mathrm{a}$ & $117,1 \mathrm{a}$ & $479,1 \mathrm{a}$ & $335,9 a$ \\
\hline C. cajan & $290,1 \mathrm{a}$ & $488,3 c$ & $106,7 \mathrm{a}$ & $343,7 b$ & $468,7 \mathrm{a}$ & $958,3 b$ \\
\hline Testemunha & $279,2 \mathrm{a}$ & $439,6 c$ & $229,1 \mathrm{~b}$ & $635,4 c$ & $843,8 \mathrm{a}$ & $2229,2 \mathrm{c}$ \\
\hline $\mathrm{CV}(\%)$ & 36,6 & 18,8 & 26,8 & 36,1 & 39,7 & 26,1 \\
\hline
\end{tabular}

Médias seguidas pelas mesmas letras nas colunas, não diferem significativamente pelo teste de Tukey ao nível de $5 \%$ de probabilidade.

Para a densidade de plantas analisadas aos 36 DAS não foi constatado diferença significativa (tabela 1). Aos 60 DAS houve diferença significativa, a $C$. spectabilis apresentou melhor desempenho na redução da densidade de plantas espontâneas, aos 36 DAS a densidade era em média de 205,7 (plantas. $\mathrm{m}^{-2}$ ) e aos 60 DAS a densidade caiu para 78,1 (plantas. $\mathrm{m}^{-2}$ ). Por sua vez a $C$. ochroleuca proporcionou um aumento quanto a densidade de plantas espontâneas, indo de 230,2 (plantas $\mathrm{m}^{-2}$ ) aos 36 DAS para 282,8 (plantas $\mathrm{m}^{-2}$ ) aos 60 DAS.

Em relação a característica de massa fresca, apenas aos 60 DAS houve diferença significativa entre os tratamentos avaliados (tabela 1). Aos 60 DAS a $C$. spectabilis e $C$. ochroleuca foram estatisticamente iguais em relação a diminuição de massa fresca de plantas espontâneas e superiores aos demais tratamentos. Com destaque para a C. spectabilis que obteve uma taxa de redução da massa fresca de $86,6 \%$ dos 36 DAS para os 60 DAS, enquanto a $C$. ochroleuca obteve uma taxa de $29,8 \%$. O C. cajan por sua vez não apresentou bom 
rendimento na diminuição de massa fresca de plantas espontâneas em relação aos demais tratamentos.

\section{DISCUSSÃO}

\section{Massa seca}

O bom desempenho da $C$. spectabilis, na redução de massa seca das plantas espontâneas, está diretamente ligado com sua capacidade de produção de biomassa, que rapidamente proporciona grande taxa de cobertura do solo, apresentando melhor sombreamento ao longo do tempo em relação a C. ochroleuca. Da Ros e Aita (1996), relataram que a diferença na cobertura do solo, pelas leguminosas é atribuída a diversos fatores, dentre eles destaca-se a própria arquitetura da planta e a velocidade com que cada espécie se desenvolve.

Analisando a (figura 1) a $C$. spectabilis foi o único tratamento que aos 60 DAS apresentou massa seca de plantas espontâneas menor que aos 36 DAS. Queiroz et al. (2008), estudando a Crotalaria juncea aos 30 dias após e emergência, encontrou média de 121,1 (g.m ${ }^{2}$ ), semelhante à descrita nesse estudo, em relação a C. spectabilis aos 36 DAS. Ferreira et al. (2018), analisando Crotalaria juncea em sistema de consórcio com Urochloa ruziziensis, obteve um bom desempenho na redução de massa seca de plantas espontâneas por metro quadrado.

Erasmo et al. (2004) verificaram que as espécies $C$. spectabilis, Sorghum bicolor, $C$. ochroleuca, Mucuna aterrima e Mucuna pruriens reduziram significativamente o número e o peso da massa seca da população das plantas espontâneas avaliadas (Digitaria horizontalis, Hyptis lophanta e Amaranthus spinosus). Araújo et al. (2007), estudando a supressão de plantas espontâneas em Miranda do Norte-MA, constatou média de biomassa de plantas espontâneas em parcelas de C. cajan de 392, 2 (g.m ${ }^{-2}$ ) aos 202 DAS e Queiroz et al. (2008), aos 30 dias após a emergência das leguminosas encontrou média de massa seca de plantas espontâneas em parcelas de C. cajan de 128,4 (g.m $\left.\mathrm{m}^{-2}\right)$, números parecidos aos observado nesse estudo aos 36 DAS e aos 60 DAS, o que pode indicar que a supressão realizada pela leguminosa $C$. cajan, pode ter pouca variação na após os 60 DAS. 


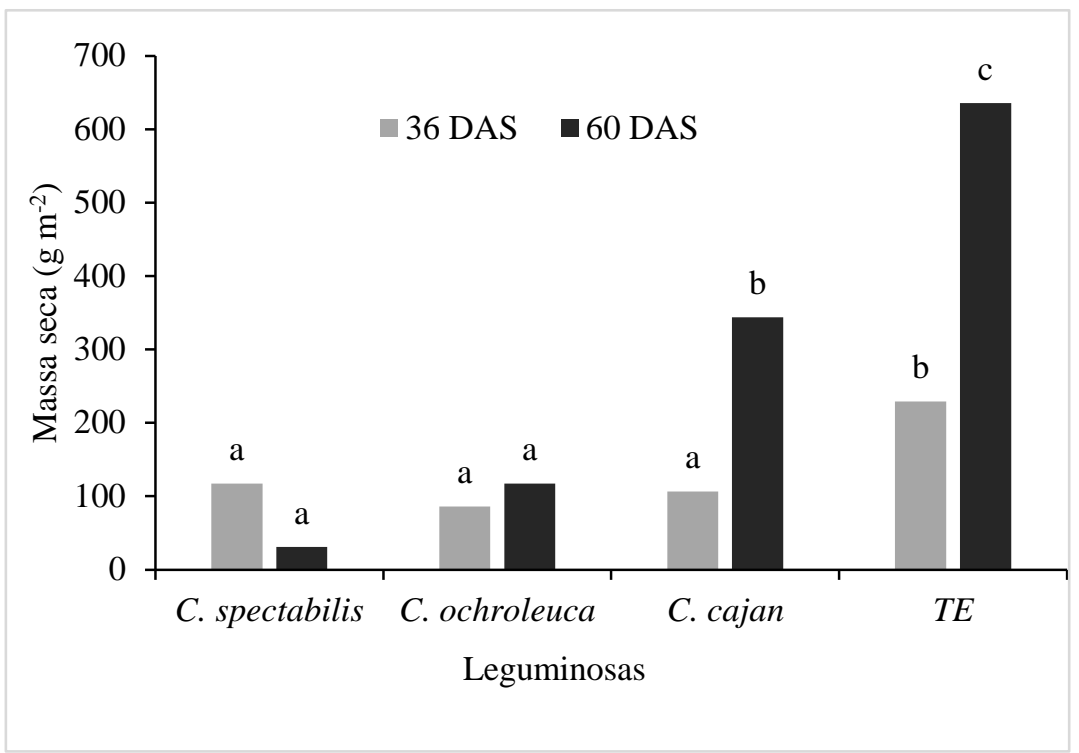

Figura 1. Massa seca de plantas espontâneas, aos 36 e 60 dias após a semeadura das leguminosas (DAS). Barras de cor igual, com letras iguais, não diferem entre si, pelo teste de Tukey, a 5\% de probabilidade. $T E$, testemunha sem leguminosa.

\section{Densidade}

A espécie $C$. cajan, apresentou números de densidade de plantas espontâneas maiores que a testemunha aos 36 DAS e aos 60 DAS, o que pode ser atribuído a sua baixa velocidade de cobertura. Por outro lado, Erasmo et al. (2004), observando a densidade de plantas espontâneas na palhada de $C$. cajan aos 30 dias após o corte do material vegetal, observou melhor eficiência em relação ao presente estudo utilizando cobertura viva.

Gomes (2014), estudando supressão de plantas espontâneas por crotalária e sorgo, confirmou a diminuição significativa na densidade de plantas espontâneas na presença de $C$. spectabilis. Soares et al. (2012) estudando Crotalaria juncea constatou que a rotação com essa cultura é eficiente na diminuição da densidade de plantas espontâneas em três sistemas de cultivos: cultivo mínimo, plantio convencional e plantio direto, tendo maior destaque no cultivo mínimo. Por sua vez, Sediyama et al. (2010) confirmou o efeito positivo da palhada de café e do bagaço de cana, na redução da densidade de plantas espontâneas. A figura 2 demonstra que assim como para a massa seca de plantas espontâneas a $C$. spectabilis foi o único tratamento que aos 60 DAS apresentou densidade de plantas espontâneas menor em comparação aos 36 DAS, o que confirma o seu potencial de supressão ao longo do tempo estudado. 


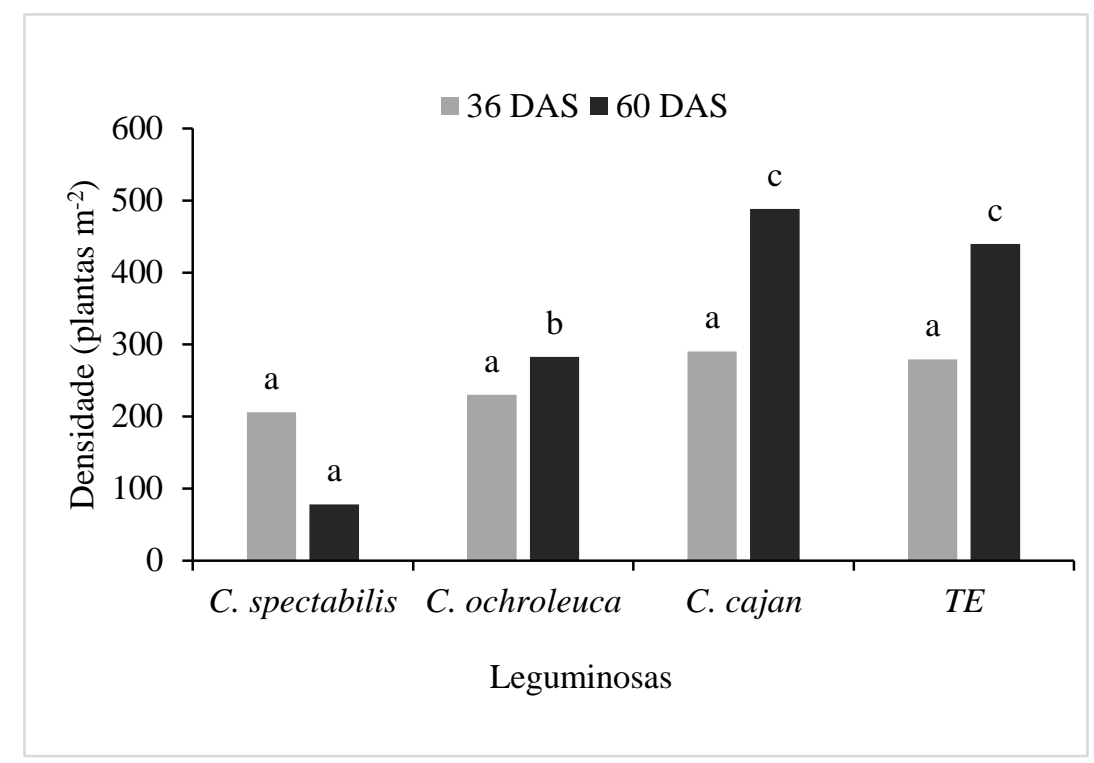

Figura 2. Densidade de plantas espontâneas, aos 36 e 60 dias após a semeadura das leguminosas (DAS). Barras de cor igual, com letras iguais, não diferem entre si, pelo teste de Tukey, a 5\% de probabilidade. $T E$, testemunha sem leguminosa.

\section{Massa fresca}

A capacidade de supressão de plantas espontâneas está diretamente ligada ao efeito alelopático, que nas leguminosas deve-se à presença de compostos químicos como taninos, alcaloides como a monocrotalina, isolada em sementes, folhas e caules de Crotalaria spectabilis (DE ALMEIDA, 1991; COSTA et al., 1995), e aminoácidos não-protéicos, como a L-Dopa identificado na mucuna (FUJII et al., 1991), capazes de influenciar o desenvolvimento de outras espécies vegetais, dependendo apenas dos seus teores nas plantas e da forma como são liberados no solo (COSTA et al., 1995).

Observando a (figura 3) é possível notar que ao longo do tempo estudado a $C$. spectabilis e a C. ochroleuca, diminuíram a massa fresca de plantas espontâneas, comportamento inverso ao demostrado pela $C$. cajan e pela testemunha. O desempenho de biomassa das duas crotalárias também é um fator favorável na supressão de massa fresca de plantas espontâneas. Segundo Teasdale et. al. (1991), isso é confirmado em seu estudo que avaliou o efeito de diversas culturas de cobertura sobre a incidência de plantas espontâneas e os melhores resultados foram encontrados com os tratamentos que produziram maior 
biomassa. Sediyama et al. (2010) obteve resultados semelhantes aos dessa pesquisa estudando plantio direto de beterraba na palha do café e bagaço de cana.

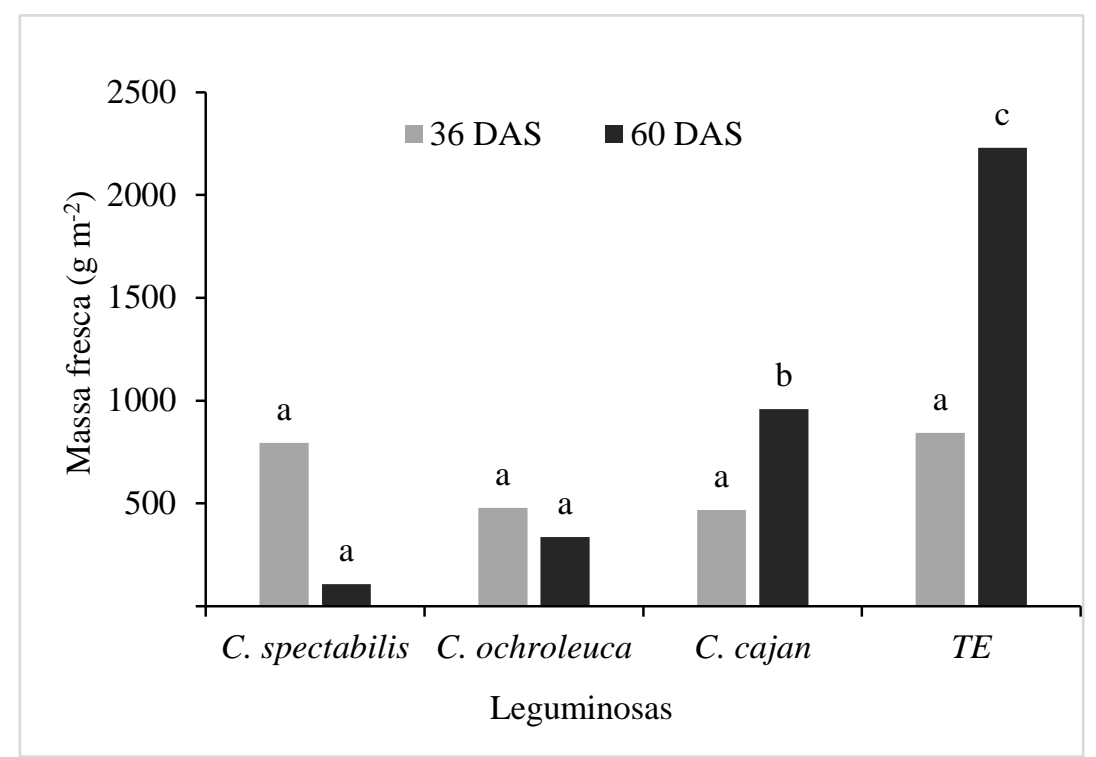

Figura 3. Massa de matéria fresca de plantas espontâneas, aos 36 e 60 dias após a semeadura das leguminosas (DAS). Barras de cor igual, com letras iguais, não diferem entre si, pelo teste de Tukey, a $5 \%$ de probabilidade. $T E$, testemunha sem leguminosa.

\section{CONCLUSÕES}

O uso de adubos verdes foi eficiente na supressão de plantas espontâneas nas condições estudas.

As espécies $C$. spectabilis e $C$. ochroleuca se mostraram alternativas vantajosas na supressão de plantas espontâneas em todas as variáveis estudadas.

\section{AGRADECIMENTOS}

Ao Benevaldo Evangelista por ter cedido à área bem como auxiliado nos procedimentos do estudo.

\section{REFERÊNCIAS}

\section{ALTIERI, M. A.; NICHOLLS, C. I. Agroecology and the search for a truly sustainable} agriculture. United Nations Environmental Programme, Environmental Training Network for Latin America and the Caribbean, 2005. 
ALVARES, C. A. et al. Köppen's climate classification map for Brazil. Meteorologische Zeitschrift, v. 22, n. 6, p. 711-728, 2013.

ARAUJO, J. C. et al. Supressão de plantas daninhas por leguminosas anuais em sistema agroecológico na Pré-Amazônia. Planta daninha, v. 25, n. 2, p. 267-275, 2007.

BOND, W.; GRUNDY, A. C. Non-chemical weed management in organic farming systems. WeedRes., v. 41, p. 283-405, 2001.

BORÉM, A.; RANGEL, P. H. N. Arroz - do plantio à colheita. Viçosa, MG: Universidade Federal de Viçosa, 242p, 2015.

COBUCCI, T. Manejo e controle de plantas daninhas em arroz de sequeiro. In: VARGAS, L.; ROMAN, E.S. (Eds). Manual de manejo e controle de plantas daninhas. Bento Gonçalves: Embrapa Uva e Vinho, 2004. p.323-336.

COSTA, A.S.V. da PESSANHA, G.G., CARVALHO, M.G. de, BRAZ FILHO, R. Identificação de substâncias secundárias presentes em leguminosas utilizadas como adubo verde. Revista Ceres, vol. 42, n. 244, p.584-598.1995.

DA ROS, C. O.; AITA, C. Efeito de espécies de inverno na cobertura do solo e fornecimento de nitrogênio ao milho em plantio direto. Revista Brasileira da Ciência do Solo, Campinas, v. 20, p. 135-140, 1996.

DE ALMEIDA, F. S. Efeitos alelopáticos de resíduos vegetais. Pesquisa Agropecuária Brasileira, v. 26, n. 2, p. 221-236, 1991.

DO NASCIMENTO, A. F.; DE MATTOS, J. L. S. Produtividade de biomassa e supressão de plantas espontâneas por adubos verdes. Agroecología, v. 2, p. 33-38, 2008. 
ERASMO, E. A. L.; AZEVEDO, W. R.; SARMENTO, R. A.; CUNHA, A. M.; GARCIA, S. L. R. Potencial de espécies utilizadas como adubo verde no manejo integrado de plantas daninhas. Planta Daninha, Viçosa-MG, v. 22, n. 3, p. 337-342, 2004.

FAVERO, C. et al. Modificações na população de plantas espontâneas na presença de adubos verdes. Pesquisa Agropecuária Brasileira, v. 36, n. 11, p. 1355-1362, 2001.

FERREIRA, A. C. de B. et al. Suppressive effects on weeds and dry matter yields of cover crops. Pesquisa Agropecuária Brasileira, v. 53, n. 5, p. 566-574, 2018.

FUJII, Y., SHIBUYA, T., YASUDA, T. L-3,4- Dihydroxyphenilalanine as an allelochemical candidate from Mucuna pruriens (L.) DC. var. utilis. Agricultural and biological chemistry, v.55, n.2, p.617-618, 1991.

GOMES, D. S. et al. Supressão de plantas espontâneas pelo uso de cobertura vegetal de crotalária e sorgo. Revista Brasileira de Agroecologia, v. 9, n. 2, p. 206-213, 2014.

LORENZI, H. Manual de identificação e controle de plantas daninhas: plantio direto e convencional. 7.ed. Nova Odessa: Instituto Plantarum, 2014. 375 p.

MALDONADO, J.A.C; OSORNIO, J.J.J.; BARRAGÁN, A.T.; ANAYA, A.L. The use of allelopathic legume cover and mulch species for weed control in cropping systems. Agronomy journal, v. 93, n.1, p.27-36. 2001.

QUEIROZ, L. R. et al. Supressão de plantas espontâneas por leguminosas anuais na cultura do milho verde, em sistema orgânico de produção. In: Embrapa Milho e Sorgo-Artigo em anais de congresso (ALICE). In: CONGRESSO NACIONAL DE MILHO E SORGO, 27.; SIMPOSIO BRASILEIRO SOBRE A LAGARTA-DO-CARTUCHO, SPODOPTERA FRUGIPERDA, 3.; WORKSHOP SOBRE MANEJO E ETIOLOGIA DA MANCHA BRANCA DO MILHO, 2008, Londrina. Agroenergia, produção de alimentos e mudanças climáticas: desafios para milho e sorgo: trabalhos e palestras.[Londrina]: IAPAR;[Sete Lagoas]: Embrapa Milho e Sorgo, 2008. 
SEDIYAMA, M. A. N. et al. Ocorrência de plantas daninhas no cultivo de beterraba com cobertura morta e adubação orgânica. Planta daninha, v. 28, p. 717-725, 2010.

SOARES, M. B. B. et al. Plantas daninhas em área de reforma de cana crua com diferentes manejos do solo e adubos verdes em sucessão. Revista Agro@ mbiente On-line, v. 6, n. 1, p. 25-33, 2012.

TEAM, RStudio et al. RStudio: integrated development for R. RStudio, Inc., Boston, MA URL http://www. rstudio. com, v. 42, 2015.

TEASDALE, J.R.; BESTE, C.E.; POTTS, W.E. Response of weeds to tillage and cover crop residue. Weed Science, Champaign, v. 39, p.195-199, 1991. 